(C) 2018 IEEE. Personal use of this material is permitted. Permission from IEEE must be obtained for all other uses, in any current or future media, including reprinting/republishing this material for advertising or promotional purposes, creating new collective works, for resale or redistribution to servers or lists, or reuse of any copyrighted component of this work in other works

\title{
Electroluminescence Imaging of PV Devices: Advanced Vignetting Calibration
}

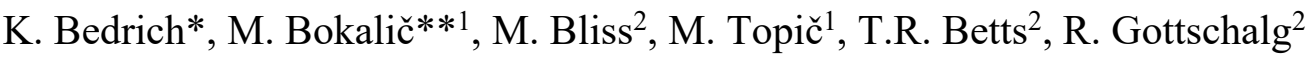 \\ ${ }^{1}$ Laboratory of Photovoltaics and Optoelectronics (LPVO), Faculty of Electrical Engineering, \\ University of Ljubljana, Tržaška cesta 25, SI-1000 Ljubljana, Slovenia \\ ${ }^{2}$ Centre for Renewable Energy Systems Technology (CREST), Loughborough University, \\ Loughborough, Leicestershire, LE11 3TU, UK \\ *Corresponding Author Tel.: +65 6567 8073, Email: karl.bedrich@nus.edu.sg \\ **Corresponding Author Tel.: +386 14786 276, Email: Matevz.Bokalic@fe.uni-lj.si
}

\begin{abstract}
Electroluminescence (EL) imaging is affected by off-axis illumination together with sensor and lens imperfections. The images' spatial intensity distribution is mainly determined by the vignetting effect. For quantitative EL imaging its correction is essential. If neglected, intensities can vary significantly $(>50 \%)$ across the image. This paper introduces and tests four vignetting measurement methods. The quantitative comparison of different methods shows that vignetting should be characterized preferably in plane by the source of the same type as the PV device to be tested. A direct PV based measurement in short distance with spatial inhomogeneity correction is proposed for general purpose vignetting characterization and vignetting-object separation using pattern recognition is proposed for precise vignetting characterization. The use of non-PV light sources for vignetting characterization can cause vignetting overcorrection and can even decrease the quality of the vignetting corrected images.
\end{abstract}

Index Terms - Electroluminescence, Vignetting, Flat Field, Calibration, Photovoltaic cells, Photovoltaic Modules.

\section{INTRODUCTION}

$\mathrm{S}$ patially resolved electroluminescence (EL) imaging of photovoltaic (PV) devices is a fast and easily applied measurement method, first proposed in [1]. However, in addition to the actual EL emission from the device under test (DUT), multiple effects related to the measurement system are superimposed in the final image as introduced in [2]. Specifically, the information in an EL image is affected by off-axis illumination, lens and sensor imperfections, which decrease the image quality.

For the EL image to represent the EL emission, these inaccuracies must be removed from the image. They can be categorized into optical aberrations and other inaccuracies such as vignetting and flare.

Optical aberrations cause light to reach the sensor at slightly shifted locations, resulting in a less sharp and more distorted image. Vignetting is caused by spatially inhomogeneous shading of the sensor by the lens, lens assembly, iris, and other optical elements. In result, light only partially reaches the sensor. This causes an erroneous image intensity distribution [3, 4], which is most often visible as a bright image center with darker corners and edges. Since the use of EL imaging is evolving to emphasize quantitative analysis, such inaccuracies must be corrected for. This paper evaluates known and newly proposed methods for measuring and correcting illuminance inhomogeneities.

These inhomogeneities can be removed by dividing every (dark current corrected) EL image taken by the vignetting image (Figure $1, I_{V i g}$ ), which describes the spatial non-uniformity of light detection.

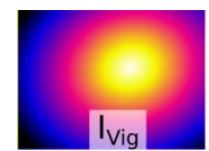

Figure 1: Example vignetting image $\left(I_{V i g}\right)$

The effect of vignetting is demonstrated in Figure 2. Row (a) shows raw EL images of the same PV device taken under the same conditions, but at different positions within the image plane.

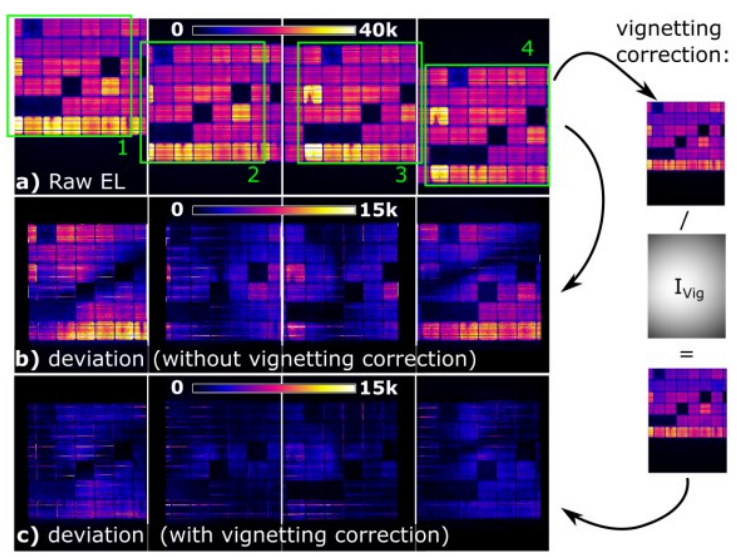

Figure 2: Intensity of (a) the same PV module imaged at different positions and corresponding intensity deviations (b) without and (c) with vignetting correction relative to the reference image 
Before further calculation, the images were background corrected [5-7], the lens distortion [8] was removed and the position of all images was unified [9]. The image with the most central position of the DUT was chosen as reference image. The absolute deviation between the reference and other EL images is shown in row (b). It reveals significant variations $(>30 \%)$.

The same deviation after vignetting correction is shown in row (c). Here, deviations are close to negligible. Noting that row (b) and (c) have the same color scale, it shows that vignetting correction is essential for reducing measurement uncertainty of quantitative EL imaging.

\section{A. Literature Review}

For astronomy based applications, Howell et al. uses the inside of a telescope dome or a dawn or dusk sky as homogenous sources for obtaining $I_{V i g}$ [10]. Kang et al. extract $I_{V i g}$ through imaging a sheet of white paper in the focal plane. There, $I_{V i g}$ is calculated as function of off-axis illumination (i.e. natural vignetting) [11]. This function is referred to as KW in the post processing Section III.

Unlike many other imaging methods, the EL method images the light source itself and not its reflection. However, homogeneous light sources that occupy the image plane and emit similar spectra to PV DUTs, are rarely available. In EL imaging literature, this homogeneity issue is bypassed as follows:

Vignetting measurement with a red liquid crystal display (LCD) at short distance and thereby out of focus of the camera is used in [12, 6]. It is reported that the emitted light at $612 \mathrm{~nm}$, may be used as an approximation for measuring cadmium telluride (CdTe) solar cells with a recombination peak at around $850 \mathrm{~nm}$ [13]. This method is used as reference (REF) in the comparison Section IV.

A similar measurement with a 'homogeneous' light source (such as rear contact solar cell) and with a defocused lens is proposed by Köntges et al. [5]. This method is excluded from the comparison because both examined camera lenses in this work were not able to blur inhomogeneities of the in-plane imaged Si modules sufficiently. Additionally, the different focus modified the optical vignetting. In consequence, the quality of images corrected with this method was worse than the other methods presented in this work. The same publication also proposed an 'angle-of-view' (AoV) fit function. This function calculates the intensity decrease from a Lambertian surface for variable aperture angles. Amongst others, this method is used to fit measured image intensities in the post processing Section III.

${ }^{1}$ https://github.com/radjkarl/imgProcessor/tree/mast er/imgProcessor/camera/flatField

\section{B. Paper organization}

The paper is organized as follows. In Section II we present four novel $I_{V i g}$ measurement methods (A-D). To improve the measurement results, different post processing methods are presented in Section III. The quality of vignetting removal of these measurement-post-processing combinations is measured and evaluated in Section IV. The work is concluded in Section V.

The code for all presented measurement and post processing methods is made public in the Python library imgProcessor ${ }^{1}$, which is embedded in dataArtist ${ }^{2}$, a graphical environment for scientific image processing.

\section{VignETTING MEASUREMENT}

This paper determines and contrasts vignetting on two module scale EL imaging systems:

- the setup at the Laboratory of Photovoltaics and Optoelectronics (LPVO) within the University of Ljubljana and

- $\quad$ the setup at the Centre for Renewable Energy Systems Technology (CREST) within the Loughborough University.

The following methods either average or correct for source inhomogeneity. All images taken were first corrected for dark current, by subtracting a background image $\left(I_{D K}\right)$ taken at the same exposure time. $[5-7,12,14]$

The quality of vignetting characterization depends on whether the optical properties of the imaged source are representative of the DUT's luminescence. Therefore, it is advisable to perform vignetting measurements in the usual measurement plane, with the same camera parameters and the same wavelength range as the DUT. Vignetting calibration quality depends on the temporal stability of the system components and measured light source. A vignetting calibration from an unsuitable light source can decrease image quality (Section IVA). The camera is considered stable after it has reached a constant operating temperature. If a PV device is chosen as source, it should be kept in forward biased excitation until its temperature stabilizes. Especially in the case of metastable PV devices, the stability of emission must be ensured.

\section{A. Direct measurement at short distance with inhomogeneity correction}

Similar to reference method REF, this method images the light source directly in front of the camera lens in order to blur out spatial inhomogeneities. However, even heavily defocused, strong inhomogeneities in a PV module (e.g. wavepattern from cell layout, see Figure 3a) remain.

${ }^{2}$ https://github.com/radjkarl/dataArtist 
Therefore a more uniform source (e.g. a commercially available LCD, see Figure $3 b$ ) can be found desirable. To the naked eye, these sources may look uniform, however an intensity difference of up to $10 \%$ was found for the same LCD source, when imaged at slightly different positions in front of the lens. An unintentionally introduced gap between source and lens also alters the result due to off-axis illumination (Figure 3c).

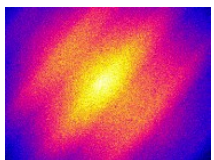

a) Thin film PV module

Figure 3: Method A, example images

Therefore method A takes a minimum of 10 ( $k=1$ to 10 ) images of the source, at different positions within the image plane and rotation angles relative to the optical axis. If in all images taken the camera sensor is homogenously illuminated, it is assumed that spatial non-uniformity averages out. However, the angular dependency of the source will still affect the result, since the source is imaged out of the image plane. The vignetting calibration image $\left(I_{V i g, A}\right)$ is then obtained from the mean along these images:

$$
\begin{gathered}
I_{V i g, A, \text { sum }}=\sum_{k=1}^{n} I_{k} \\
I_{V i g, A}=\frac{I_{V i g, A, \text { sum }}}{\max \left(I_{V i g, A, \text { sum }}\right)}
\end{gathered}
$$

The sum operator operates over all given images by the same pixel index and therefore returns an image. Finally, the image is normalized to obtain $I_{V i g, A}$.

\section{B. Discrete spot average}

Method A measures vignetting directly in front of the camera. Hence, the direction of light rays differs to that in standard measurement setup and can cause erroneous results. In order to measure vignetting in plane with the DUT, the calibration source is placed directly on a DUT mounted in the imaging plane. This aligns the optical axis between camera and source as it will be for actual measurements. The source size should not exceed $10 \%$ of the image plane, to allow multiple images of it to be taken at different positions (Figure 4a). Using the following algorithm, a set of points $(P)$ used to fit a vignetting function (Section III) is obtained: For every taken image $\left(I_{k}\right)$ :

1. Select foreground by thresholding the image using Otsu's method [15] ( $\left.T_{\text {Otsu }}\right)$ to obtain the mask $\left(M_{k}\right)$ :

$$
M_{k}=I_{k}>T_{\text {Otsu }}
$$

2. Filter small areas and select the largest masked/selected coherent pixel group $\left(M_{k, \max }\right)$.

3. Extract the center of gravity $\left(x_{k}, y_{k}\right)$ and average intensity $\left(z_{k}\right)$ in the selected area:

$$
\begin{aligned}
x_{k,} y_{k} & =\frac{1}{S} \iint M_{k, \text { max }} d x d y \\
z_{k} & =\operatorname{mean}\left(I_{k}\left[M_{k, \text { max }}\right]\right)
\end{aligned}
$$

The averaged intensities of all pixel groups $\left(z_{k}\right)$ are used to fit a vignetting equation (Figure $4 \mathrm{~b}$ ):

$$
I_{V i g, B}=\operatorname{FIT}\left(z_{k}\right)
$$

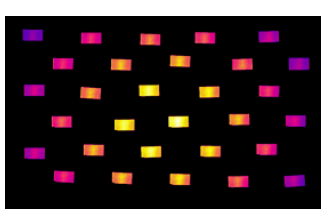

a) Image overlay from 31 individual images $\left(I_{k}\right)$ Figure 4: Method B example results using a mobile phone screen as light source

This method combines the simplicity of method A with in-plane imaging. In doing so, it trades uncertainty due to out-of-plane imaging for uncertainty of the light source temporal stability. Additionally, using only a small number of images/locations to fit the whole image adds to the correction uncertainty. For calibration sources other than DUT, errors due to wavelength and angular differences remain.

\section{Vignetting-Object separation from discrete steps}

This method corrects for the DUT's inhomogeneity through measuring average intensities of the same device at different predefined positions.

This can be, for example, single cells of a c-Si based module. Multiple EL images of the DUT at different discrete locations within the image plane are taken (Figure 5a). In the shown example, the image plane is divided into a $13 \times 13$ grid of $120 \mathrm{~mm}$ squares. The DUT is a $6 \times 12$ cell c-Si module. For each imaged position, the signal within each grid square is averaged (Figure $5 \mathrm{~b}$ ). The result is assigned to a layer of the image stack $\left(S_{\text {Grid }}\right)$ incorporating the DUT and the image plane (Figure 5c). Areas imaging the background are excluded using a threshold condition (Equation 2). In the example, $(n=10)$ images were used (only three shown for clarity). From the average over all grid images, an initial vignetting image $\left(I_{v i g, 0}\right)$ is built (Figure $5 f$ ). From the same stack, a new stack containing only DUT cells $\left(S_{D U T}\right)$ is created and cell positions $(P)$, 
needed to index the same areas in $S_{\text {Grid }}$ and $S_{D U T}$, are extracted (Figure 5c,d).

$I_{\text {vig, } C}$ is then calculated iteratively with the iteration step $(i)$ and the image index $(k)$ via:

1. Extract the average DUT cell values:

$$
I_{\text {avDUT, }, i}=\frac{\sum_{k=1}^{k=n}\left(\frac{S_{D U T}[k]}{I_{v i g, i}[P[k]]}\right)}{n}
$$

2. Create next vignetting map from average ratio between the DUT stack and the average cell values:

$$
I_{\text {Vig }, i+1}[P[c]]=\frac{\sum_{k=1}^{k=n}\left(\frac{S_{D U T}[k]}{I_{\text {avDUT }, i}}\right)}{n}
$$

3. Check iteration criterion using given threshold value $\left(T_{\text {otsu }}\right)$ : If $\sum\left(I_{V i g, i+1}-I_{V i g, i}\right)>T_{\text {otsu }}$, then go to step 1, else normalize the results:

$$
I_{V i g, C}=\frac{I_{V i g, i+1}}{\max \left(I_{V i g, i+1}\right)}
$$

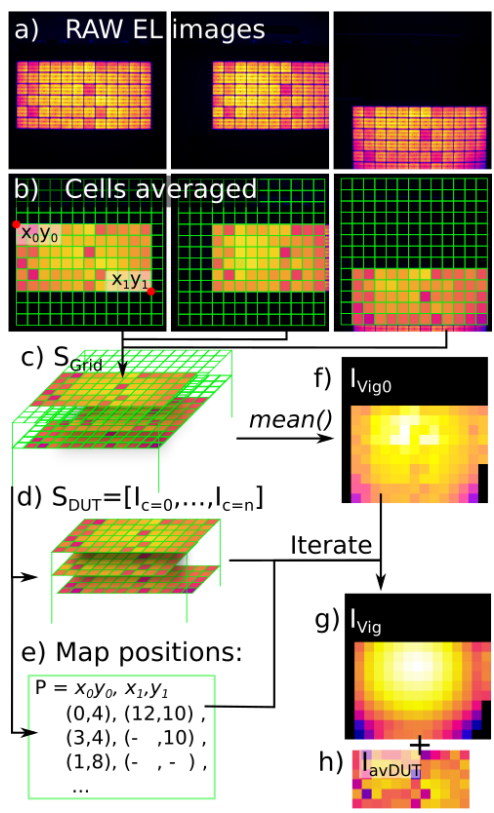

Figure 5: Method C scheme to obtain vignetting image

This method measures vignetting in plane and allows the same DUT for calibration and measurement. However, its result is a low-resolution grid, which needs to be up-scaled to camera resolution. Missing or incorrect areas within $I_{v i g, C}$ are corrected through post processing (Section III). This method can be adjusted easily for different grid cell (and physical PV cell) sizes, but needs precise placement of the DUT in order not to affect the measured averages.

\section{Vignetting-object separation using pattern recognition}

This method builds on top of method C. Instead of averaging DUT intensities within a predetermined grid, the translation and rotation of the DUT is detected within each EL image $\left(I_{k}\right)$ relative to a reference image using pattern recognition. This has two advantages:

- The DUT position within the image can be chosen randomly, provided a good overall coverage of the measurement plane is achieved.

- The resulting vignetting image has the same resolution as the input images (e.g. $3000 \times 4000$ vs $13 \times 13$ via method C).

$I_{V i g, D}$ is obtained as follows (Figure 6):

1. The DUT is imaged at different positions and (if needed - not required) angles within the image plane. A reference image $\left(I_{r e f}\right)$ showing most of the DUT (preferably without rotation) is selected for later pattern recognition. The DUT is masked (green box) using Equation 2.

2. An initial vignetting array is calculated from the moving maximum of each individual image. Reasonable improvements to image quality are achieved for image areas that are covered by at least three DUT images. The resulting array is smoothed to reduce inhomogeneities.

3. a) Each image taken is transformed to the same perspective as the reference image. For this purpose, key points of both images are matched using ORB ('Oriented FAST and Rotated BRIEF'[16]), implemented in the free computer vision library OpenCV [8].

b) Overall vignetting is extracted from the individual vignetting image from each rectified DUT image via inverse perspective transformation.

4. Each rectified DUT image (a) is divided by each rectified vignetting array (b), respectively. The result is averaged to obtain the first vignetting corrected DUT image (c).

5. Each image (a) is divided by (c) to recover each individual vignetting array. All arrays are perspective transformed into the original position using the same process as in step 3 .

Steps 4 and 5 are repeated until the difference between the last two vignetting arrays falls below a given iteration threshold.

6. To obtain $I_{V i g, D}$, empty areas are synthesized and inhomogeneities smoothed out in post processing (Section III). 


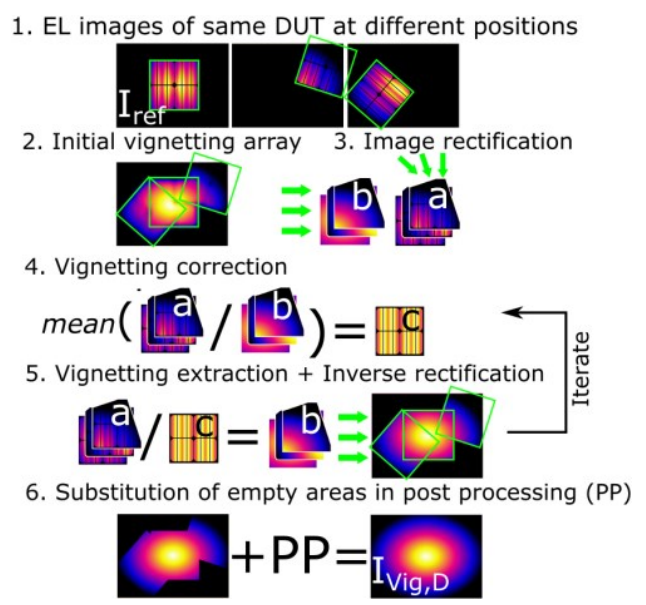

Figure 6: Calculation of a vignetting image using pattern recognition in an iterative process $($ method $\mathrm{D})$

\section{POST PROCESSING}

All vignetting measurement methods result in either a vignetting image (methods $\mathrm{A}, \mathrm{D}$ ) or discrete points (methods $\mathrm{B}$ and $\mathrm{C}$ ). The vignetting images may include noise and artefacts and may not cover the whole imaging area. Conversely, the discrete points are only a sample of small areas in the overall imaging area. Thus, depending on the vignetting measurement method, a degree of post processing is often required to obtain a usable vignetting correction image. Since the effect of vignetting is continuous, the following filters or functional approximations can be used to obtain a smooth vignetting correction image from the measured RAW image:

- KW: Simplified Kang-Weiss vignetting (Equation 9))

- AoV: Angle-of-view equation from [5]

- POLY: $2^{\text {nd }}$ order 2D polynomial fit

These methods are applied in two fashions:

- = (replace): Every image pixel is replaced with a fitted value.

- + (repair): Only empty areas are infilled with fitted values. For +POLY this also includes high gradient areas.

Finally, common filters are applied to $+\mathrm{KW}$ :

- $\quad+\mathbf{K W}$,Gauss: Image convolution with Gaussian kernel.

- $\quad+\mathbf{K W}$,median: Every image pixel is replaced with the median of the surrounding pixels.

Both image filters have a kernel size of 5\% relative to the image width. The simplified Kang-Weiss vignetting equation from [17] is:

$$
\begin{gathered}
I_{v i g}=A \cdot(1-\alpha \cdot d) \\
A=\frac{1}{\left(1+\left(\frac{d}{f}\right)^{2}\right)^{2}} \\
d=\sqrt{\left(x-x_{0}\right)^{2}+\left(y-y_{0}\right)^{2}}
\end{gathered}
$$

$$
\begin{array}{ll}
f \ldots & \text { focal length }[\mathrm{px}] \\
x_{0}, y_{0} \ldots & \text { image centre }[\mathrm{px}] \\
\alpha \quad \ldots & \text { geometric vignetting factor }[-]
\end{array}
$$

Figure 7 illustrates example results of the post processing methods applied to vignetting measurements with method D on two similar samples at different measurement setups. The upper part of the RAW vignetting image (a) was not characterized and remains black due to spatial constraints for the setup at LPVO. Similarly, a vertical stripe remains black in RAW vignetting image (b) for the setup at CREST. All post processing methods filled the black areas. Both RAW images contain remaining patterns, seen as vertical and horizontal lines. These are caused by misalignment errors after perspective transformation in method D. When post processing is applied in ' + ' (repair) fashion, these artefacts remain, but when the '=' (replace) fashion is used they disappear. +POLY also smoothens out these high gradient variations. Gauss and median filtering of $+\mathrm{KW}$ additionally blurs or even removes these artefacts.

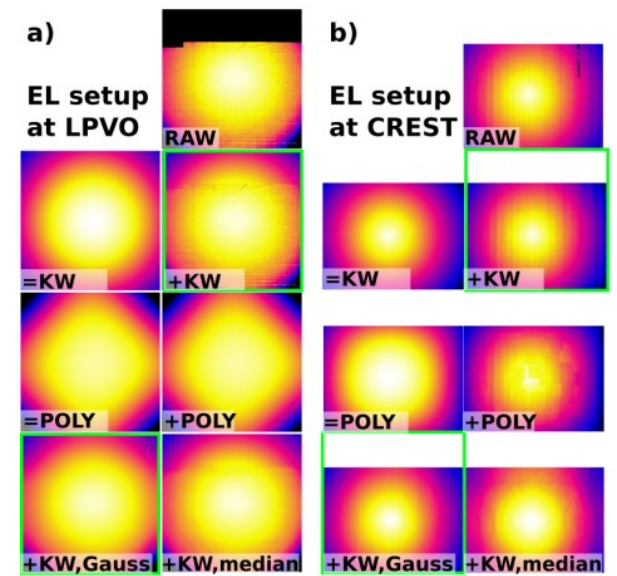

Figure 7: Comparison of different post processing methods applied to two similar samples on two different EL setups; raw image obtained from method E; values scaled $50-100 \%$; proposed post processing methods highlighted green

\section{Vignetting And Post Processing Methods COMPARISON}

The following algorithm to compare vignetting measurement methods and applicable post processing algorithms is similar to the presented method D. In the same way, multiple images of the DUT are taken at different (random) positions within the image plane (Figure 8a). Images are rectified using the same feature-matching algorithm (b). From all rectified images $\left(I_{k}\right)$, an image average $\left(I_{\text {avg }}\right)$ is built (c). A relative image difference $\left(D_{k}\right)$ is then calculated for each $I_{i}$ as follows:

$$
D_{k}=\frac{I_{k}-I_{a v g}}{I_{a v g}}
$$


Misalignment errors after perspective transformation can cause high magnitudes along high gradient variation, like cell edges and busbars in $I_{k}$. To remove this influence, a cell average is performed. It results in a lower resolution image (e) which only contains vignetting effects, in case the DUT signal is temporally stable. A root-meansquare error (RMSE) of all cell-averaged image differences finally returns the relative error, caused by residual vignetting $(R)$. This approach is used to obtain the inherent setup vignetting error $\left(R_{\text {raw }}\right)$ from uncorrected EL images and the residual vignetting error $\left(R_{i, j}\right)$ from all corrected images. Vignetting correction was performed for all measurement methods $(i)$ and all post processing methods $(j)$.

From all RMSE pairs $\left(R_{\text {raw }}, R_{i, j}\right)$ the vignetting correction quality $\left(Q_{i, j}\right)$ is then determined as follows:

$$
Q_{i, j}=\frac{R_{\text {raw }}-R_{i, j}}{R_{\text {raw }}}
$$

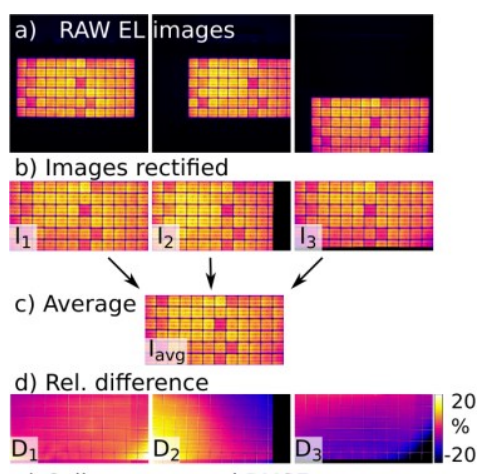

e) Cell average and RMSE

$$
\mathrm{R}_{\text {Raw }}\left(\mathrm{D}_{\mathrm{D}_{2}}\right)=23 \%
$$

Figure 8: Scheme of calculating error from residual vignetting

Image setup parameters for both cases are shown in Table 1.

Table 1: Image setup parameters of compared cases

\begin{tabular}{lll}
\hline Setup & CREST & LPVO \\
\hline \hline Camera name & Sensocam HR-830 & FLI ML16803 \\
Focal length & $25 \mathrm{~mm}$ & $50 \mathrm{~mm}$ \\
Aperture & $\mathrm{f} / 2.8$ & $\mathrm{f} / 2.8$ \\
\hline
\end{tabular}

\section{A. Vignetting correction}

A comparison of different vignetting and post processing method combinations is shown in Figure 9 for the two different setup-module combinations.

a) CREST - large PV: c-Si, small PV: c-Si cell

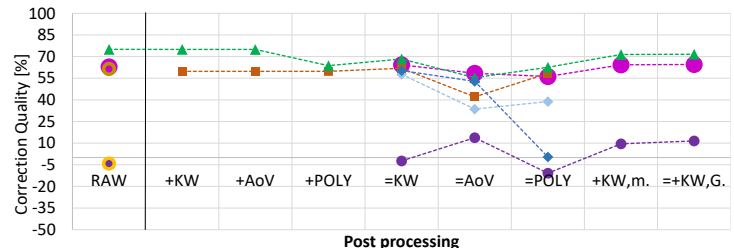

b) LPVO - large PV: c-Si HIT, small PV: c-Si cell

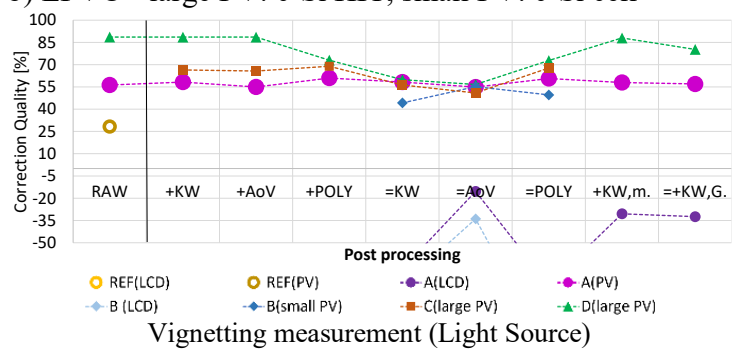

Figure 9: Correction quality $\left(Q_{i, j}\right.$, see Equation (13)) for two different EL setup-module combinations; Note that the values are discrete and the lines are only to guide the eye.

Along the $\mathrm{x}$-axis different post processing method are compared. The evaluated measurement method-light source combinations are shown as colored plots. Note that the values are discrete and the lines are only to guide the eye.

For method REF with light sources LCD and PV $(\mathrm{REF}(\mathrm{LCD} / \mathrm{PV})$ ) an average of three randomly chosen close distance images was built. For methods A through D, 10 to 20 EL images were analyzed.

In order to find the best suitable measurement method, boxplots, based on data shown in Figure 9, were generated (Figure 10). Vignetting correction quality ranges from below $-110 \%$ to $90 \%$.

It can be seen, that methods using a PV device as a light source provided better vignetting correction. Method $\mathrm{D}(\mathrm{PV})$ corrected vignetting best. It is followed by methods $\mathrm{C}(\mathrm{PV}), \mathrm{A}(\mathrm{PV})$, and $\mathrm{B}(\mathrm{PV})$ which still provide good correction. On the other side, methods using an LCD screen as a light source often even decreased image quality due to vignetting overcorrection.

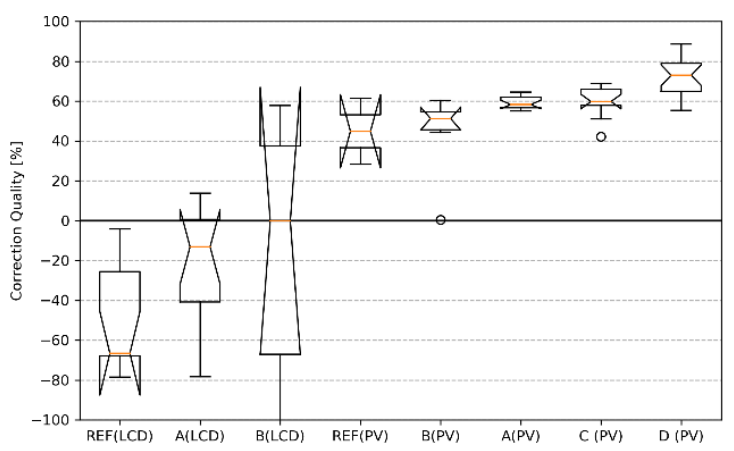

Figure 10: Vignetting method comparison from values shown in Figure 9

A direct comparison of vignetting corrected images using REF(LCD) and D(PV) (Figure 11d,e) underlines the different correction qualities. 


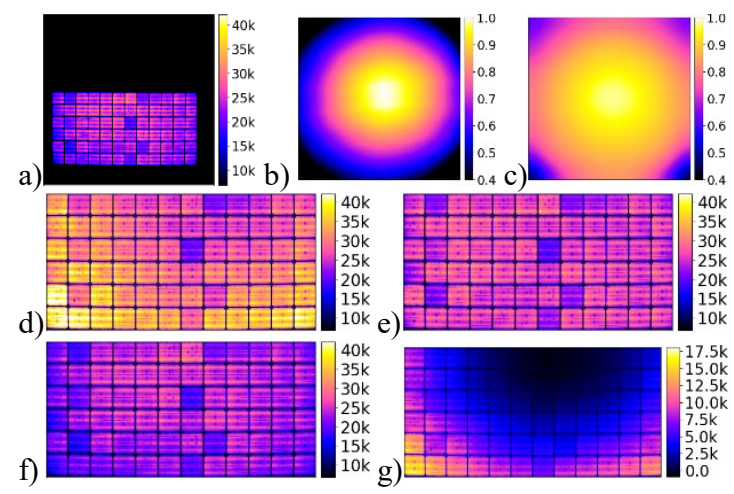

Figure 11: Influence of vignetting correction quality on a) EL image using method b) REF(LCD) and c) D(PV); d-f) EL image after perspective- and vignetting correction using method d) REF(LCD) and e) D(PV);

f) no vignetting correction;

g) Image difference (d-e); all images taken at LPVO

The reason for significant source dependency lies in different angular radiation patterns and in different wavelength ranges. Since natural vignetting is mainly caused by off-axis illumination, it is highly angular dependent. Similarly, light of different wavelengths is reflected and refracted differently and again causes different vignetting effects. Both differences, radiation pattern and wavelength inequality result in $25 \%$ lower vignetting intensities along the image edge for $\mathrm{A}(\mathrm{LCD})$ in comparison to $\mathrm{A}(\mathrm{PV})$ (Figure 12).

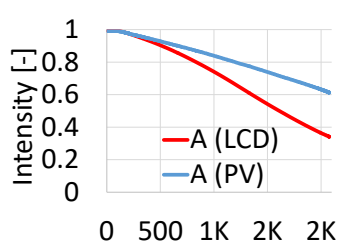

$\begin{array}{lllllllll}0 & 500 & 1 \mathrm{~K} & 2 \mathrm{~K} & 2 \mathrm{~K}\end{array}$

a) CREST Radius [px]

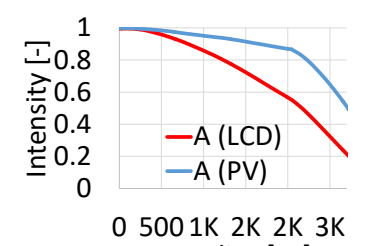

b) LPVO Radius [px]
Figure 12: Radial average of $I_{V i g, A}$ measured using an LCD screen and a PV module (c-Si)

In comparison to method REF (both LCD and PV), the method A (both LCD and PV) produced equal or better results. This shows that taking more images of the same light source at close proximity at different locations and orientation is beneficial. This is due to averaging of inhomogeneous illumination, varying angular distribution and temporal instability.

The correction quality of method B(LCD) varies strongly, even results in correction qualities below $100 \%$ (Figure 10 ). Such bad results are caused by the LCD used with method B at the LPVO setup (Figure $9 b)$. Figure 13 presents the vignetting corrected overlay of 25 images used to measure vignetting via method B. Should the source be homogeneous and the vignetting correction appropriate, these images should show no vignetting, but that is not the case. This is caused by: 1 . Residual vignetting due to the use of a non-PV light source (Figure 13a). 2. superposition of temporal instability, and inhomogeneous angular emission of the used LCD light source (Figure 13b). This example shows how important the selection of the light source is, and how much it can affect the otherwise sound method B.

a)
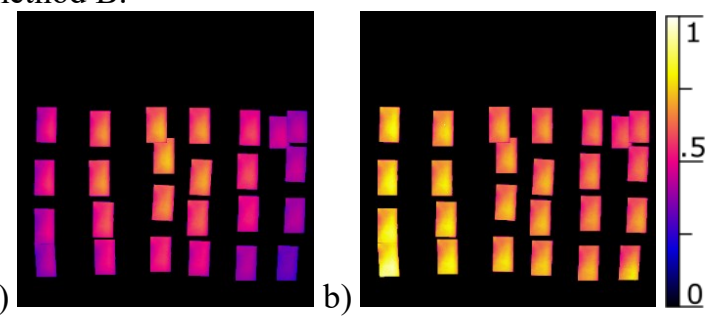

Figure 13: Sum of all images used to measure method B at LPVO setup after vignetting correction using a) method $\mathrm{A}(\mathrm{PV})$ and $\mathrm{b}) \mathrm{A}(\mathrm{LCD})$. Scale relative to maximum intensity.

\section{A. Post processing}

The effect of different post processing methods on vignetting correction is visible in the correction quality plot along the $\mathrm{x}$-axis in Figure 9. The detailed improvement due to post processing relative to RAW vignetting images is shown in Figure 14. The results indicate that $+\mathrm{KW}$, Gauss increased the image quality. However, in most cases at the first glance post processing decreased image quality. In comparison of both functional fits, KW resulted in better vignetting correction that AoV. The effect of post processing on the actual image is presented with cross-sections through the centers of the images in Figure 15. It can be seen that both $=\mathrm{AoV}$ and $=\mathrm{KW}$ caused a vignetting overcorrection towards the image edges (blue plots, Figure 15). The reason for this is that both AoV and KW fit natural vignetting. However actual vignetting is also influenced by lens and iris shading. A polynomial fit (POLY) can be useful in such cases, where the measured vignetting does not match natural vignetting. Measurement artefacts can also be reduced using image filters. The use of median-based filters is discouraged, since it can result in image intensity steps in the vignetting image (red plot, Figure 15), however the use of blur filters (e.g. Gaussian blur - yellow dashed plot, Figure 15) is beneficial.

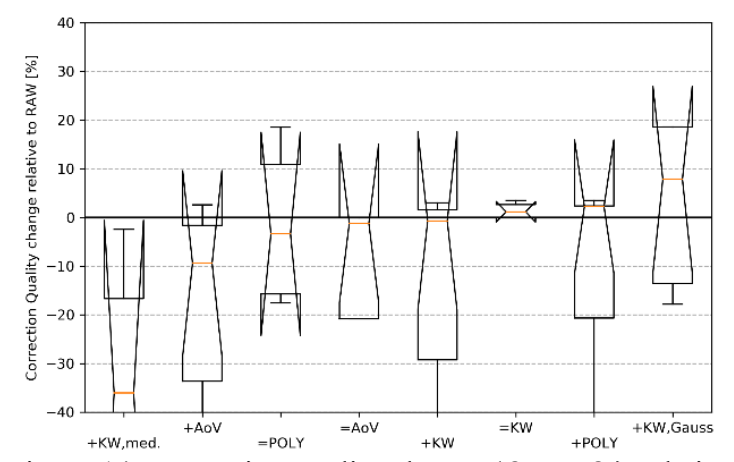

Figure 14: Correction quality change $\left(Q_{i, j}-Q_{i}\right)$ relative to RAW $\left(Q_{i}\right)$ of different post processing methods on the correction quality from values shown in Figure 9 


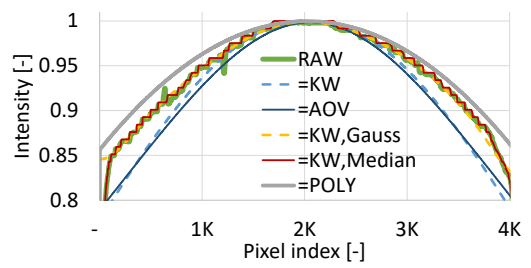

Figure 15: Horizontal line plots through image center of LPVO results from method E (Figure 7a)

Quantitative evaluation of post processing quality by the used quality parameter $\left(Q_{i, j}\right)$ only evaluates average intensity deviations and not local inhomogeneities and artefacts in vignetting images. The main purpose of post processing is to smooth local inhomogeneities present in RAW vignetting images. Therefore, a small quantitative quality decrease can be acceptable if, in exchange, the postprocessing routine returns a smooth and artefact free image.

The decision of the most suitable post processing method depends on the quality of the RAW image and the shape of measured vignetting. To select the best method, it is suggested to measure and compare their correction quality (Figure 14).

\section{CONCLUSION}

Vignetting significantly influences pixel intensity of photovoltaic luminescence images. Therefore, vignetting correction is essential. In this paper we focused on several measurement and post processing methods, suitable for vignetting removal in luminescence images.

The most prevalently used vignetting measurement method REF images a "homogeneous" light source in close proximity to the camera lens. It provides unsatisfactory results, especially when nonhomogeneous LCD screens are used as a light source. In general, a light source similar to the actual DUT should be used to characterize vignetting. Even the DUT itself can be used when appropriate methods are used.

The simplest method presented in this paper (method A: direct measurement in short distance with inhomogeneity correction) upgrades the established method REF by acquiring further images of the same source in different orientations to cancel out its inhomogeneity. The resulting vignetting correction quality is close to the best methods when a light source, similar to the DUT is used. Therefore, due to simplicity and applicability, method $\mathrm{A}(\mathrm{PV})$ is proposed for general vignetting characterization.

Method D (vignetting-object separation using pattern recognition) is technically the most advanced method. It images the actual DUT at random positions in image plane. Amongst all tested methods, it produced the best results. It is proposed for precise luminescence characterization.
It is suggested to fill empty areas within the measured vignetting image with a Kang-Weiss functional fit. To smoothen out erroneous pixels, the use of a Gaussian filter is proposed. This work focusses on in-plane vignetting and neglects the specific camera setup. The effect of image plane angle and -distance, camera aperture and exposure time will be covered in future work.

\section{REFERENCES}

[1] T. Fuyuki, H. Kondo, T. Yamazaki, Y. Takahashi, and Y. Uraoka, "Photographic surveying of minority carrier diffusion length in polycrystalline silicon solar cells by electroluminescence," Appl. Phys. Lett., vol. 86, no. 26, p. 262108, 2005.

[2] M. Bokalič and M. Topič, Spatially resolved characterization in thin-film photovoltaics. Springer, 2015.

[3] R. Szeliski, Computer Vision: Algorithms and Applications, Springer. Springer London, 2010.

[4] M. Bokalič, J. Raguse, J. R. Sites, and M. Topič, "Analysis of electroluminescence images in small-area circular CdTe solar cells," J. Appl. Phys., vol. 114, no. 12, p. 123102, 2013.

[5] M. Köntges, M. Siebert, and D. Hinken, "Quantitative analysis of PV-modules by electroluminescence images for quality control," Proc. 24th ..., 2009.

[6] D. Hinken, K. Ramspeck, K. Bothe, B. Fischer, and R. Brendel, "Series resistance imaging of solar cells by voltage dependent electroluminescence," Appl. Phys. Lett., vol. 91, no. 18, p. 182104, 2007.

[7] M. Bliss, X. Wu, K. Bedrich, T. R. Betts, and R. Gottschalg, "Spatially and Spectrally Resolved Electroluminescence Measurement System for PV Characterisation," in IET$R P G$, accepted 2015.

[8] G. Bradski and A. Kaehler, Learning OpenCV: Computer vision with the OpenCV library. O'Reilly, 2008.

[9] K. G. Bedrich, M. Bliss, T. R. Betts, and R. Gottschalg, "Electroluminescence Imaging of PV Devices: Camera Calibration and Image Correction," IEEE PVSC-43, 2016.

[10] S. B. Howell, Handbook of Ccd Astronomy. 2006.

[11] S. B. Kang and R. Weiss, "Can we calibrate a camera using an image of a flat textureless lambertian surface?," Eur. Conf. Comput. Vison, vol. 2, pp. 640-653, 2000.

[12] T. Potthoff, K. Bothe, U. Eitner, D. Hinken, and M. Köntges, "Detection of the voltage distribution in photovoltaic modules by electroluminescence imaging," Prog. Photovoltaics Res. Appl., vol. 18, no. 2, pp. 100-106, Mar. 2010.

[13] M. Topič, J. Raguse, K. Zaunbrecher, M. Bokalič, and J. R. Sites, "Electroluminescence of Thin Film Solar Cells and PV Modules - Camera Calibration," in 26th European Photovoltaic Solar Energy Conference and Exhibition, 2011, pp. 2963-2966.

[14] F. J. J.L. Crozier, E.E. van Dyk, "High Resolution Spatial Electroluminescence Imaging of Photovoltaic Modules," Vor. Nelson Mand. Metrop. Univ., 2009.

[15] N. Otsu, "A threshold selection method from gray-level histograms," IEEE Trans. Syst. Man. Cybern., vol. 9, no. 1, pp. 62-66, 1979.

[16] E. Rublee, V. Rabaud, K. Konolige, and G. Bradski, “ORB: An efficient alternative to SIFT or SURF," Proc. IEEE Int. Conf. Comput. Vis., pp. 2564-2571, 2011.

[17] S. Lin, "Single-Image Vignetting Correction," 2006 IEEE Comput. Soc. Conf. Comput. Vis. Pattern Recognit. - Vol. 1, vol. 1, pp. 461-468, 2006. 